\section{You won't believe the gleam!}

Trycare Ltd, the UK's fastest growing dental supplier, is the UK distributor for Eve surgical polishing tips and strips. Manufactured in Germany from premium grade materials, Eve polishing tips and strips enable clinicians to achieve the most natural lustre and shine on their restorations whatever they are made from.

The special geometry of Diacomp Plus Twist polishers enables clinicians to polish any surface with just one polisher, resulting in a high shine and significant time saving. Offering extraordinary durability, surface structure preservation and reduced heat generation, they can be autoclaved in excess of 25 times.

Diacomp Plus Occluflex is Eve's unique occlusal polisher. It has been designed to achieve high-quality results on otherwise difficult-to-access occlusal surfaces, the twisted filaments reaching right down to th deepest fissures. They produce a high shine without the need for polishing pastes.
Both are used in a simple two-step process. First, the pink pre-polisher is used to smooth the restoration after using a gross anatomy trimmer. Then, the grey fine polisher is used to produce the final high shine finish that really makes restorations gleam. Perfect for use with Tokuyama spherical particle composites, which deliver their own exceptional lustre and finish!

For further information visit the Trycare website, www.trycare.co.uk, email dental@ trycare.co.uk, contact your local Trycare representative or call 01274885544 .

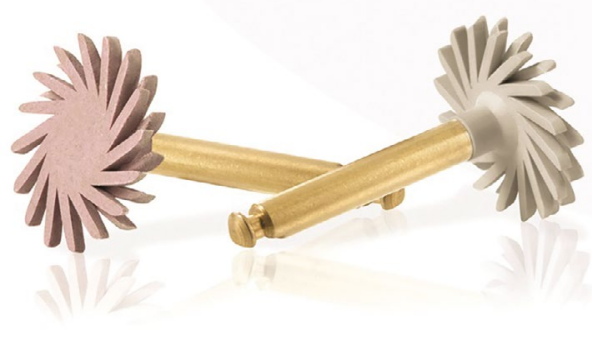

\section{Dental CPD study tours 2022}

Jon Baines has been creating successful study tours for 20 years, combining professional visits, meetings and talks with full and fascinating cultural itineraries. Each tour is led by a dedicated tour leader to ensure the journey provides insight and runs smoothly. Dental study tours in 2022 include:

\section{Dental Study Tour to Ecuador and the Galapagos Islands - ONE SPACE LEFT 19-31 August 2022}

Join tour leader, Dr Ashok Sethi, and see dentistry through the prism of Ecuador, a land of enormous cultural and natural diversity. This tour encapsulates the great variety to be found within this compact country and includes a Galapagos cruise, alongside a full programme of professional visits.

\section{Pierre Fauchard Academy Convocation 9-16 September 2022}

The 2022 Pierre Fauchard Academy Induction Ceremony for new Fellows will take place on 10 September 2022 at the Royal College of Surgeons of Edinburgh.

Join Dr Derek Mahony, UK Regent for the PFA, on a five-night post-Convocation tour to the highlands and islands of Scotland.

vacuum autoclave have been developed to effectively take handpieces through the sterilisation process.

Utilising a thermally efficient copper chamber that gives faster, more efficient cycles compared to stainless steel counterparts plus enhanced thermodynamic drying, these autoclaves ensure handpieces are better protected from corrosion, discolouration and recontamination, with the added benefit of providing an ongoing reduction in instrument and autoclave maintenance costs.

NSK is world renowned for its innovative development of precisionengineered dental handpieces. Its equally innovative, high-performance decontamination equipment helps practices maintain handpieces in peak condition, whilst also effectively and efficiently reprocessing them for safe and reliable use, time after time.

Find out more about NSK's wide range of decontamination equipment at mynsk. co.uk/decontamination/.

\section{Dental Study Tour to Japan 23 October-3 November 2022}

This tour takes you to the heart of both historic and modern Japan while exploring Japanese approaches to dentistry. Travel with Professor Phillip Dowell and visit a prestigious dental school and private dental clinics, meet Japanese dentists and experience the culture and history of Japan.

Partners are very welcome.

For more details: www.jonbainestours.com 02072239485 info@jonbainestours.co.uk.

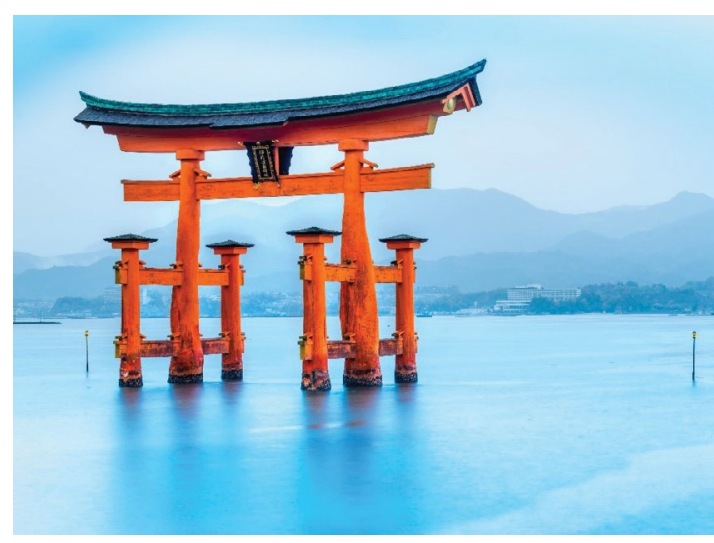

